\title{
Meal pattern validation: associations of meal size and meal timing with glucose concentrations in a population-based cohort
}

Meal patterning encompassing time, quantity and frequency of eating has been associated with diet quality, cardiovascular risk factors and coronary heart disease ${ }^{(1)}$. In observational studies, underreporting is common and might be time-of-day dependent ${ }^{(2)}$. Underreporting may bias associations between meal patterns and disease. Glucose concentrations have circadian variation corresponding to food intake ${ }^{(3,4)}$. We aimed to validate reported meal patterns by associating meal size with glucose concentrations over the day.

The Norfolk-based European Prospective Investigation into Cancer and Nutrition (EPIC-Norfolk) recruited 25,636 men and women, aged 39-79 y from GP practices between 1993-1998 ${ }^{(5)}$. At a health visit, anthropometry was measured and non-fasting blood samples were collected (08:00-19:00); serum glucose concentrations were analysed $(n=18,631)$. Participants using glucose and lipid lowering medication were excluded as well as those who reported $<4$ days, illness or nightshifts in their 7 -day diet diary (7dDD). The pre-structured 7dDD had eight recording sections: before breakfast (BB), breakfast (B), midmorning (MM), lunch $(\mathrm{L})$, tea (T), dinner (D), evening (E) and 'unknown time' (U). We calculated mean reported energy intake (MJ/d) for each section, representing 'meal size'. Analysis of covariance was adjusted for: daily energy intake (DEI), hour of blood sampling, hours fasted, eating frequency, season, sex, age, physical activity, smoking, alcohol, education and BMI $(\mathrm{N}=15,506)$. Adjusted means of glucose (Y-axis) were graphed by hour of blood sampling (X-axis) for zero, $25^{\text {th }}, 50^{\text {th }}$ and $75^{\text {th }}$ centiles of average meal size (lines). The significance of the interaction between meal size and sampling time was determined by the F-test $(P<0.05)$.

Mean (SD) DEI was $9.53(2.10)$ and $7.23(1.58) \mathrm{MJ} / \mathrm{d}$ in men and women respectively (with 4.0, 0.3 and $0.2 \%$ skipping B, L, D respectively). Mean daily glucose was 4.23 (1.49) and $4.16(1.32) \mathrm{mmol} / \mathrm{L}$ respectively, with approximately $0.5 \mathrm{mmol} / \mathrm{L}$ interval between mean peak and trough over the day $(P<0.001)$. Significant interactions between blood sampling time and lunch size were observed $(P<0.001)$, but not for breakfast or dinner.

Glucose concentrations measured at morning appointments had no dose-response association with breakfast, whereas such associations were observed for afternoon and lunch size, in addition to afternoon insulin resistance ${ }^{(3)}$. Evening blood samples were lacking. In this free-living population, meals were not iso-caloric, but represent gradual increments of meal sizes over the day (the latter more clearly observed for triglycerides ${ }^{(6)}$ ). Verifying meal skipping, and therefore meal frequency, may help elucidate meal patterndisease associations.
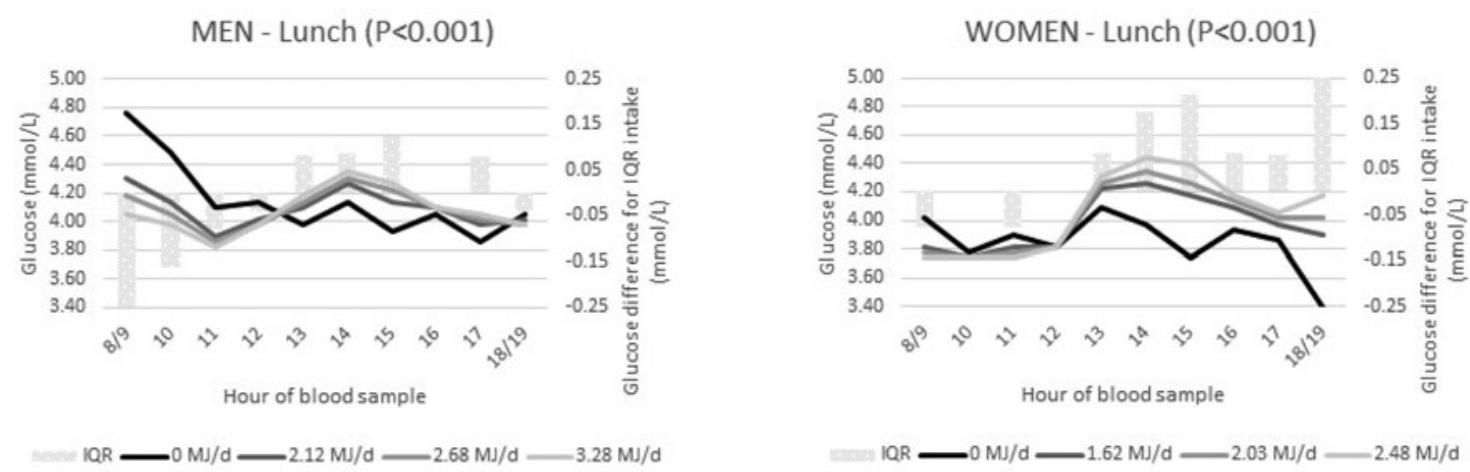

1. St-Onge M-P, Ard J, Baskin ML, et al. (2017) Circulation 135, e96-e121.

2. Gemming L \& Ni Mhurchu C (2016) Eur. J. Clin. Nutr. 70, 640-641.

3. Morgan L, Arendt J, Owens D, et al. (1998) J. Endocrinol. 157, 443-451.

4. Kanaley JA, Heden TD, Liu Y, et al. (2014) Br. J. Nutr. 112, 1484-93.

5. Day N, Oakes S, Luben R, et al. (1999) Br. J. Cancer 80 Suppl 1, 95-103.

6. Lentjes MAH, Griep LMO, Keogh RH, et al. (2017) Proc. Nutr. Soc., vol. 76, p. E129. 